\title{
Fluoroscopic balloon dilation for early jejunojejunostomy obstruction after gastrectomy with roux-en- $Y$ reconstruction: a case series of three patients
}

Teppei Kamada ${ }^{1 *}$, Hironori Ohdaira ${ }^{1}$, Sojun Hoshimoto ${ }^{1}$, Satoshi Narihiro ${ }^{1}$, Norihiko Suzuki ${ }^{1}$, Rui Marukuchi ${ }^{1}$, Hideyuki Takeuchi ${ }^{1}$, Masashi Yoshida', Eigoro Yamanouchi ${ }^{2}$ and Yutaka Suzuki ${ }^{1}$

\begin{abstract}
Background: Small bowel obstruction after gastrectomy with Roux-en-Y reconstruction (R-Y reconstruction) is not a rare complication. However, patients who need re-operation for this complication have a high rate of postoperative complications.

We report a case series of three patients who underwent fluoroscopic balloon dilation (FBD) for early jejunojejunostomy obstruction (JJO) after gastrectomy with Roux-en-Y reconstruction (R-Y reconstruction).

Case presentation: Three patients were referred to our hospital for surgery for gastric cancer. Robot-assisted distal gastrectomy with D2 lymph node dissection and antecolic R-Y reconstruction were performed in two patients, and robotassisted total gastrectomy with D1+ lymph node dissection and antecolic R-Y reconstruction was performed in one patient. The jejunojejunostomy was created as a side-to-side anastomosis using a linear $45-\mathrm{mm}$ stapler. The entry hole was closed with a knotless barbed suture, and serosal-muscle layer suture reinforcement with an absorbable suture was performed at the jejunojejunostomy.

Subsequently, all the patients were diagnosed with JJO by computed tomography and upper gastrointestinal series. The average time to $\mathrm{JJO}$ from gastrectomy was 5 days (range 2-7); initial clinical symptoms were vomiting in all three cases, with simultaneous upper abdominal pain in one case. We successfully performed FBD in all three cases after unsuccessful conservative treatment using an ileus tube. The clinical symptoms improved soon after FBD, and all the patients were able to avoid re-operation. The average period to FBD from JJO was 10 days (range 4-14). The average procedure time was 46 min (range 29-68), and the average duration to oral intake from FBD was 4 days (range 2-5). The average duration of hospital stay after FBD was 12 days (range 9-15). There were no complications in any of the cases.
\end{abstract}

Conclusion: FBD might be a feasible procedure to avoid surgery for early small bowel obstruction after gastrectomy with RY reconstruction.

Keywords: Gastrectomy, Early small bowel obstruction, Fluoroscopic balloon dilation, Barbed suture

\footnotetext{
* Correspondence: teppei0911show@yahoo.co.jp

${ }^{1}$ Department of Surgery, International University of Health and Welfare

Hospital, 537-3, Iguchi, Nasushiobara, Tochigi 329-2763, Japan

Full list of author information is available at the end of the article
}

\section{Springer Open}

(c) The Author(s). 2020 Open Access This article is licensed under a Creative Commons Attribution 4.0 International License, which permits use, sharing, adaptation, distribution and reproduction in any medium or format, as long as you give appropriate credit to the original author(s) and the source, provide a link to the Creative Commons licence, and indicate if changes were made. The images or other third party material in this article are included in the article's Creative Commons licence, unless indicated otherwise in a credit line to the material. If material is not included in the article's Creative Commons licence and your intended use is not permitted by statutory regulation or exceeds the permitted use, you will need to obtain permission directly from the copyright holder. To view a copy of this licence, visit http://creativecommons.org/licenses/by/4.0/. 


\section{Introduction}

Small bowel obstruction (SBO) after gastrectomy with Roux-en-Y reconstruction ( $\mathrm{R}-\mathrm{Y}$ reconstruction) is not a rare complication. Early small bowel obstruction (ESBO) within the first 30 postoperative days after the reconstructive surgery can be caused by technical problems, such as kinking, narrowing, or acute angulation of the anastomosis, while late SBO can occur secondary to internal hernia or intra-abdominal adhesions [1, 2].

Most patients diagnosed with ESBO after R-Y reconstruction are observed conservatively and do not require surgical intervention [1-4]. However, some patients need re-operation under general anesthesia for both diagnosis and treatment when their situation does not improve, and these cases usually have a high rate of postoperative complications $[5,6]$.

Recently, endoscopic or fluoroscopic balloon dilation has been performed to avoid re-operation for ESBO or Crohn's disease, as it is minimally invasive and preserves intestinal length $[7,8]$.

We report three cases in which fluoroscopic balloon dilation (FBD) was successfully performed to avoid reoperation for early jejunojejunostomy obstruction (JJO) after gastrectomy with $\mathrm{R}-\mathrm{Y}$ reconstruction.

\section{Case presentation}

Case 1

A 65-year-old man was referred to our hospital for surgery for advanced gastric cancer. Robot-assisted distal gastrectomy with D2 lymph node dissection and antecolic R-Y reconstruction was performed. The jejunojejunostomy was performed via minilaparotomy through an umbilical incision. The jejunojejunostomy was created $40 \mathrm{~cm}$ distal to the gastrojejunal anastomosis, as a side-to-side anastomosis using a linear 45-mm stapler (Autosuture, Covidien, Mansfield, MA, USA). The entry hole was closed with a knotless barbed suture (V-Loc ${ }^{\mathrm{TM}} 180$, Covidien, Mansfield, MA, USA). Serosal to muscle layer suture reinforcement was performed at the jejunojejunostomy using a 3-0 Vicryl suture (Ethicon, Somerville, NJ). Petersen's defect and the jejunojejunal mesenteric defect were also closed with a 3-0 Vicryl suture during the surgical procedure. The pathological diagnosis was poorly differentiated adenocarcinoma, T4a, N3a, M0, stage IIIB.

The patient initially made good progress after the operation and started oral intake on postoperative day 1 . However, on postoperative day 6 , he complained of upper abdominal pain and vomiting. Computed tomography (CT) scans showed signs of jejunal dilation between the gastric remnant and jejunojejunostomy without strangulation. Insertion of an ileus tube for decompression and bowel rest was performed, but with no improvement. On postoperative day 10, FBD was performed. The procedure time was $68 \mathrm{~min}$. On the day following FBD, his clinical symptoms improved. Oral intake was recommenced 4 days after FBD, and he was discharged 9 days after FBD.

He was followed up for 30 days and experienced no symptoms or other complications during this period.

\section{Case 2}

A 75-year-old man was referred to our hospital for surgery for advanced gastric cancer. Robot-assisted distal gastrectomy with D2 lymph node dissection and antecolic R-Y reconstruction were performed. The jejunojejunostomy was created similarly as in case 1 . The pathological diagnosis was moderately differentiated adenocarcinoma, T3, N1, M0, stage IIB.

On postoperative day 2 , he complained of vomiting. CT scans showed signs of jejunal dilation between the gastric remnant and jejunojejunostomy without strangulation. Insertion of an ileus tube for decompression and bowel rest was performed, but with no improvement in his symptoms. Hence, FBD was performed on postoperative day 14 . The procedure time was $29 \mathrm{~min}$. His clinical symptoms improved on the day following FBD. Oral intake was commenced 5 days after FBD, and he was discharged 11 days after FBD.

He experienced no symptoms or other complications during the 60 days follow-up period.

\section{Case 3}

A 79-year-old man was referred to our hospital for surgery for early gastric cancer. Robot-assisted total gastrectomy with D1+ lymph node dissection and antecolic R-Y reconstruction were performed. The jejunojejunostomy was created similarly as in case 1 . The pathological diagnosis was moderately differentiated adenocarcinoma, T1b, N0, M0, stage IA.

On postoperative day 7 , he complained of vomiting. CT scans showed signs of jejunal dilation between the esophagus and jejunojejunostomy without strangulation (Fig. 1a, b). Insertion of an ileus tube for decompression and bowel rest was performed, but without symptom improvement. Subsequently, FBD was performed on postoperative day 21. The procedure time was $42 \mathrm{~min}$. His clinical symptoms improved on the day following FBD, and oral intake was commenced 2 days after FBD. He was discharged 15 days after FBD.

The patient experienced no symptoms or other complications during the follow-up period of 150 days after FBD.

The characteristics of the three patients with jejunojejunostomy obstruction are described in Table 1.

\section{FBD procedure}

Before FBD, the ileus tube was confirmed to be positioned just proximal to the stenosis. The location and length of the stenosis were identified by injection of 


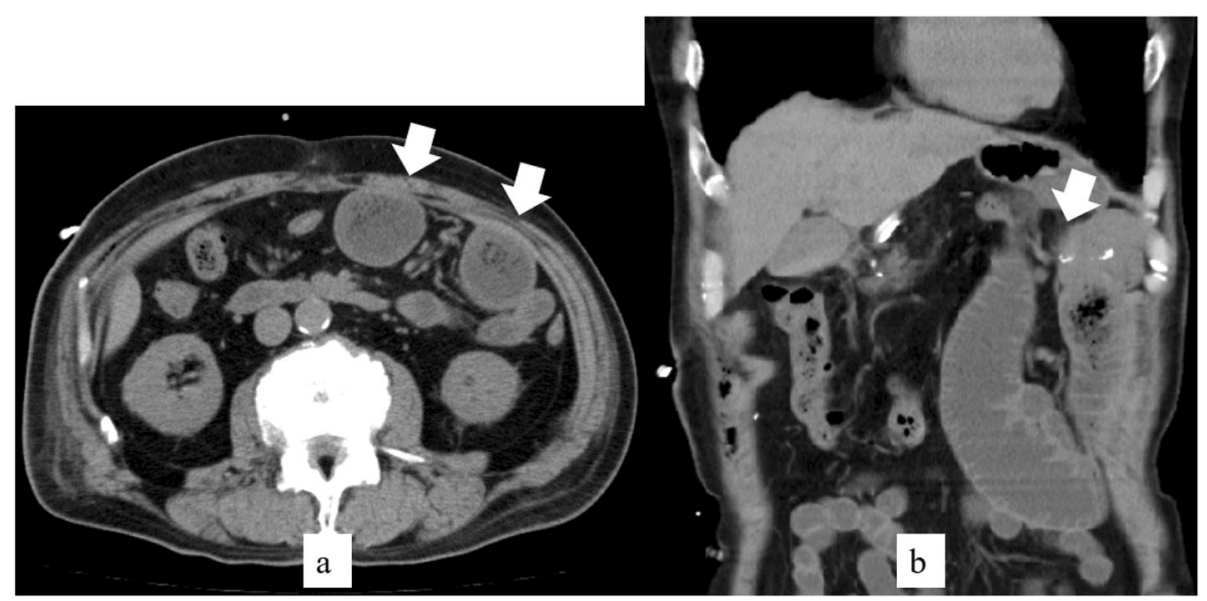

Fig. 1 a, b CT scan imaging of jejunojejunostomy obstruction (imaging of case 3). a Dilation of the elevated jejunum (arrows). b Obstruction of the jejunojejunostomy (arrow)

contrast medium through the ileus tube (Fig. 2). The appropriate size of the balloon required was determined based on the severity of stenosis.

Flunitrazepam, $0.4 \mathrm{mg}$, was injected intravenously with the patient in the supine position.

The procedure was performed under constant fluoroscopic control (Fig. 3).

A 0.035 -in, 260-cm-long stiff-angled hydrophilic guidewire (Radifocus, Terumo, Tokyo, Japan) was inserted through a nasal ileus tube and positioned over the location of the stenosis (Fig. 4a).

Next, a wire-guided balloon dilation catheter (CRE balloon catheter, Boston Scientific, Natick, MA, USA) was inserted up to the site of the stenosis. The balloon was gradually inflated with diluted water-soluble contrast medium under continuous fluoroscopic control until the stenosis completely disappeared, the inflation being maintained for 3 min thereafter (Fig. 4b, c). Then, diluted water-soluble contrast medium was injected through the catheter, and improvement of the stenosis was confirmed. Finally, the balloon catheter was removed, and an ileus tube was inserted through the guidewire to prevent re-stenosis after confirming that it could be passed through the site of stenosis (Fig. 4d). Postoperatively, the ileus tube was removed after confirming improvement of abdominal findings and bowel evacuation.

\section{Discussion}

The overall reported incidence of SBO after laparoscopic gastrectomy with R-Y reconstruction is $2.6-3.6 \%[9,10]$. Half of the SBOs after R-Y reconstruction occur in the early postoperative period [2]. Although the definition of ESBO varied among investigators, it typically includes any clinically significant and radiologically proven partial or complete obstruction at the jejunojejunostomy site occurring within the first 30 postoperative days $[3,4]$.

ESBO is caused by several factors, such as kinking, narrowing, or acute angulation of the anastomosis, for which conservative treatment is inadequate and the patient needs re-operation in the form of either an open or laparoscopic procedure.

However, since early postoperative adhesions are often very dense and highly vascular, laparoscopic exploration often requires conversion to open laparotomy. Significant comorbidity caused by bowel resection or re-R-Y

Table 1 Characteristics of the three patients with jejunojejunostomy obstruction after robot-assisted gastrectomy with Roux-en-Y reconstruction

\begin{tabular}{|c|c|c|c|c|c|c|c|c|c|c|}
\hline Case & $\begin{array}{l}\text { Age } \\
\text { (years), } \\
\text { sex }\end{array}$ & $\begin{array}{l}\text { Gastrectomy } \\
\text { Reconstruction }\end{array}$ & $\begin{array}{l}\text { Pathological } \\
\text { stage }\end{array}$ & $\begin{array}{l}\text { Duration (days) } \\
\text { (gastrectomy- } \\
\text { JjO) }\end{array}$ & Symptoms & $\begin{array}{l}\text { Duration } \\
\text { (days) (JJO- } \\
\text { FBD) }\end{array}$ & $\begin{array}{l}\text { FBD time } \\
\text { (minutes) }\end{array}$ & $\begin{array}{l}\text { Duration } \\
\text { (days) (FBD- } \\
\text { oral intake) }\end{array}$ & $\begin{array}{l}\text { Hospital stay } \\
\text { (days) (FBD- } \\
\text { discharge) }\end{array}$ & Complications \\
\hline$\# 1$ & $65, M$ & $\begin{array}{l}\text { RADG/D2 } \\
\text { R-Y }\end{array}$ & $\begin{array}{l}\text { IIIB } \\
\text { (T4aN3aM0) }\end{array}$ & 6 & $\begin{array}{l}\text { UAP, } \\
\text { vomiting }\end{array}$ & 4 & 68 & 4 & 9 & None \\
\hline \#2 & $75, M$ & $\begin{array}{l}\text { RADG/D2 } \\
\text { R-Y }\end{array}$ & IIB (T3N1M0) & 2 & Vomiting & 12 & 29 & 5 & 11 & None \\
\hline \#3 & $79, \mathrm{M}$ & $\begin{array}{l}\text { RATG/D1+ } \\
\text { R-Y }\end{array}$ & $\begin{array}{l}\text { IA } \\
\text { (T1bNOMO) }\end{array}$ & 7 & Vomiting & 14 & 42 & 2 & 15 & None \\
\hline
\end{tabular}

RADG robot-assisted distal gastrectomy, RATG robot-assisted total gastrectomy, $R-Y$ Roux-en-Y reconstruction, UAP upper abdominal pain, JJO jejunojejunostomy obstruction, FBD fluoroscopic balloon dilation 


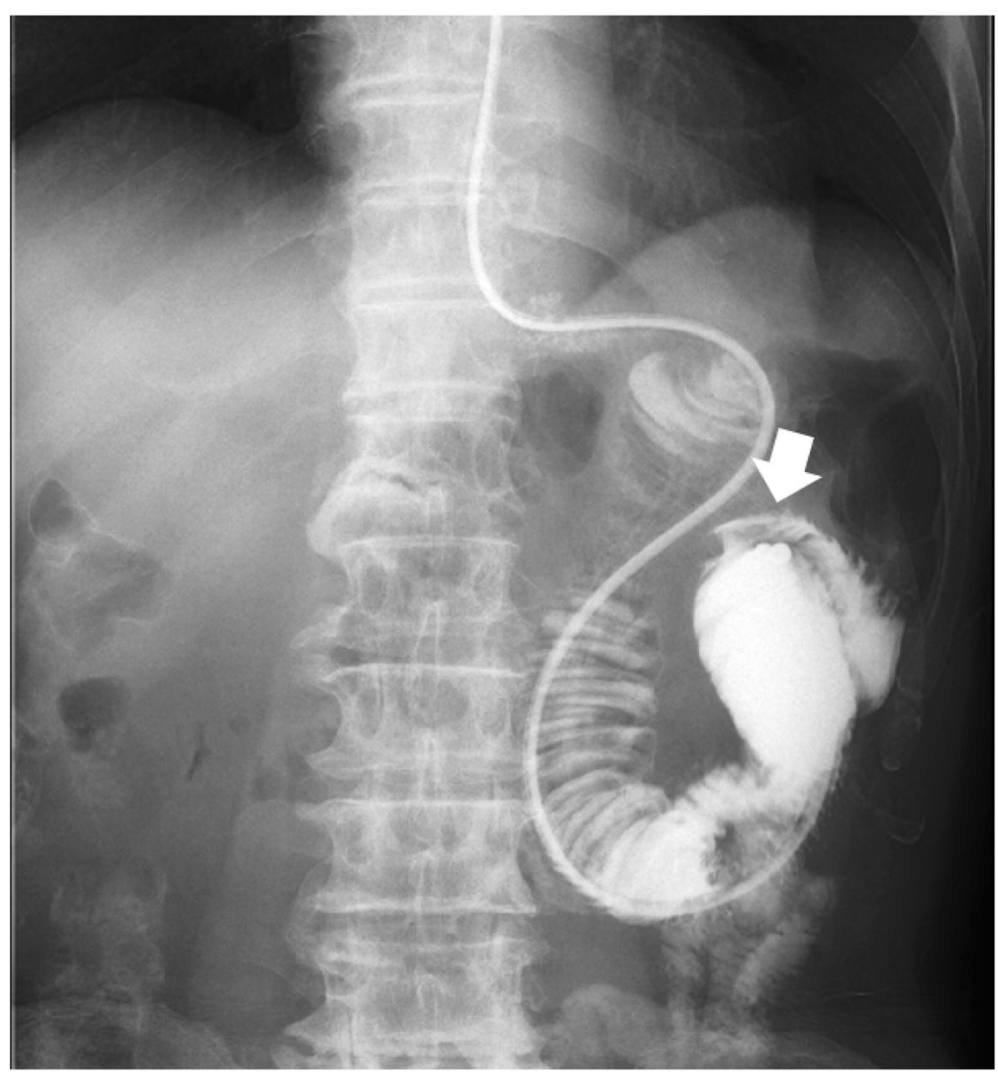

Fig. 2 Upper gastrointestinal series through the ileus tube. Stenosis of the jejunojejunostomy (arrow)

reconstruction also presented in at least half of the patients in previous studies $[5,6]$.

Recently, endoscopic or fluoroscopic balloon dilation has been shown to be a feasible procedure to avoid reoperation for ESBO or Crohn's disease, as it is minimally invasive and preserves intestinal length $[7,8]$.
Additionally, it has the potential to delay the need for surgery and might be an alternative to surgery in patients who are not candidates or who wish to avoid or postpone surgery. Balloon dilation could also have many advantages over re-operation, including the absence of the need for general anesthesia, less pain, faster return

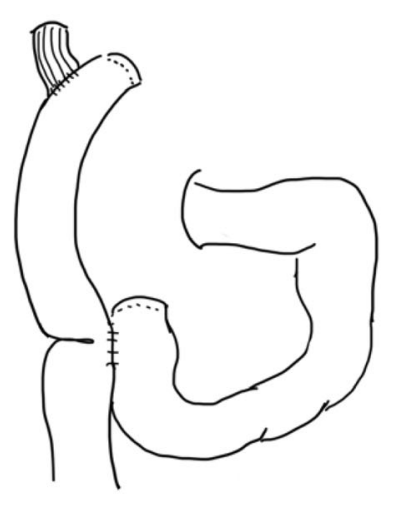

a

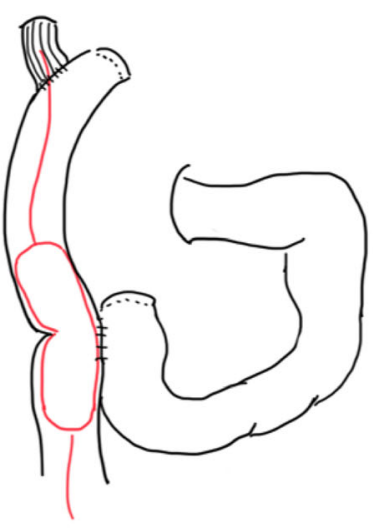

b

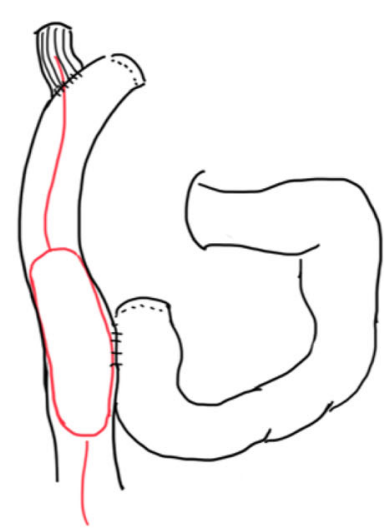

c

Fig. 3 a, b, c Schema of fluoroscopic balloon dilation 


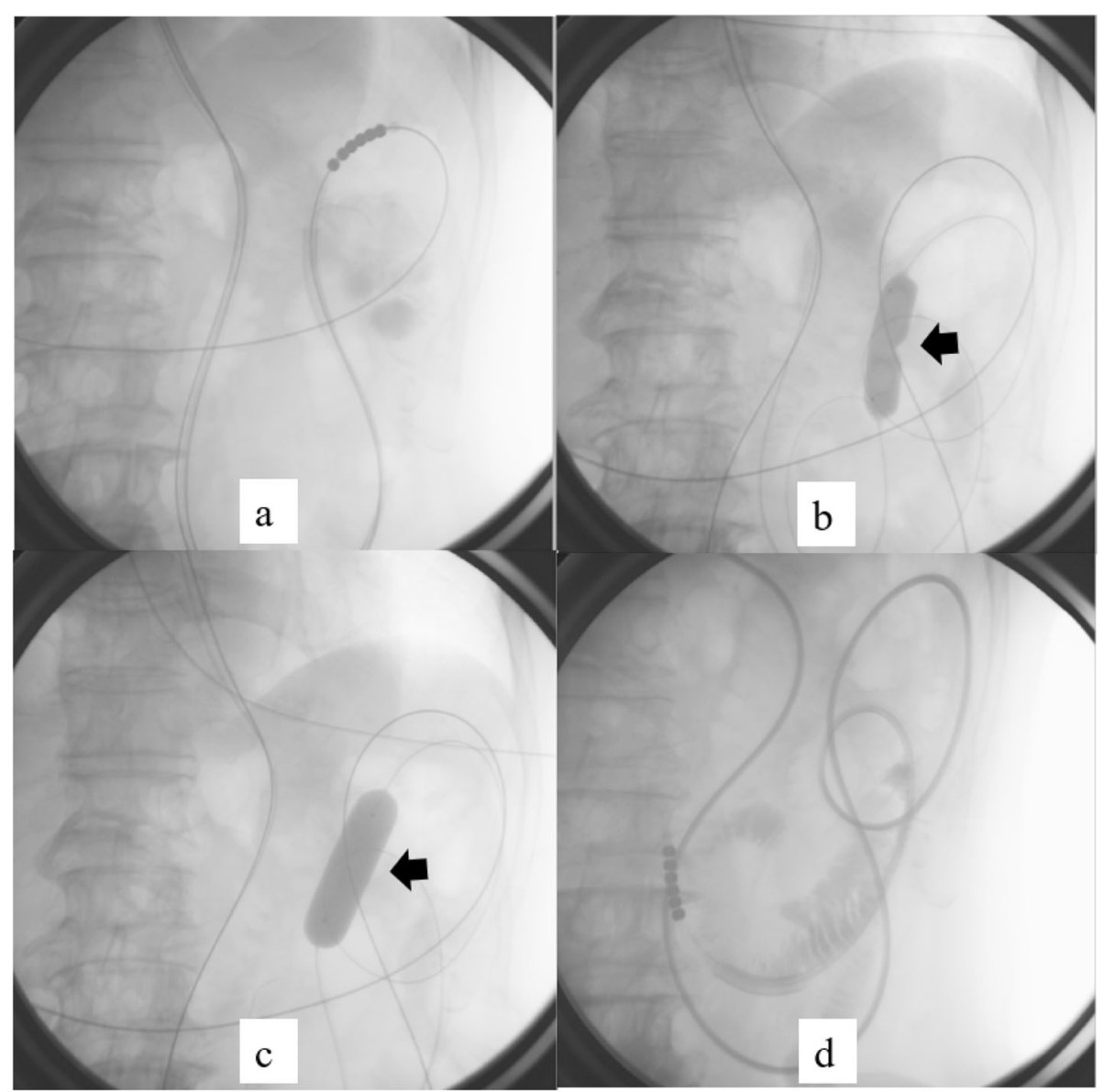

Fig. 4 a, b, c, d Fluoroscopic balloon dilation procedure (imaging of case 3). a Radiograph showing insertion of the guidewire up to the site of stenosis. b Radiograph showing the "waist" caused by stenosis of the jejunojejunostomy (arrow). c Radiograph showing disappearance of the "waist" after balloon dilation (arrow). d Radiograph showing that the ileus tube could be passed across the site of stenosis

of bowel function, shorter hospital stay, fewer complications, lower cost, and no risk of further adhesion formation $[7,8]$.

Additionally, FBD has several advantages over endoscopic balloon dilation. Although endoscopic balloon dilation is very useful for the treatment of proximal $\mathrm{SBO}$, double-balloon enteroscopy is necessary to reach the stenosis in cases of distal SBO, which is difficult to insert. Further, not all institutions have double-balloon enteroscopy systems. FBD, on the other hand, can be easily performed for distal SBO. Another advantage is that FBD is more comfortable and less invasive for patients than endoscopic balloon dilation because of the size of the catheter device.

Tsuao et al. suggested that FBD might be safe and effective for treating postoperative non-anastomotic strictures of the proximal small bowel. In their study, of 44 patients, technical success with FBD was achieved in 39 (88.6\%) patients, and the clinical success rate was $87.2 \%$. There were no major complications directly related to FBD [7].

Baars et al. reported a systematic review of 13 studies, including a total of 310 patients, in whom double-balloon enteroscopy for small bowel strictures was performed. As a result, surgery was avoided in $80 \%$ of the patients. After the first dilatation, $46 \%$ were treated with re-dilatation and only $17 \%$ required surgery. The complication rate was $4.8 \%$ per patient and $2.6 \%$ per dilatation. This included five patients with perforations, three patients with acute pancreatitis, one patient who suffered from hemorrhage that required blood transfusions, three patients with hyperamylasemia, and three patients in whom the complications were unknown [8].

Evaluation of the surgeries performed at our hospital showed that JJOs occurred in 1.1\% (4/364) patients after gastrectomy with R-Y reconstruction (including LDG, LTG, RADG, RATG) (Fig. 5). 


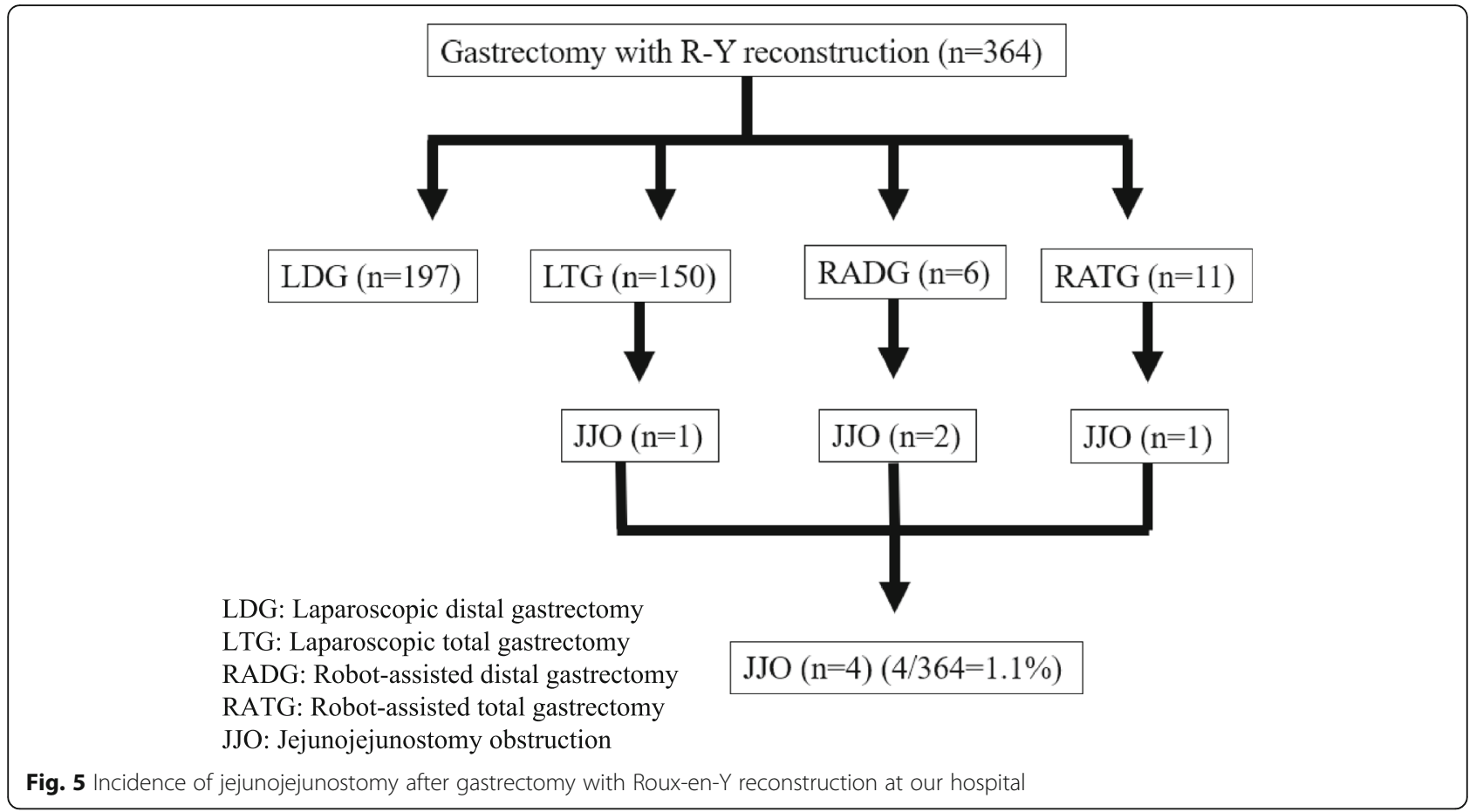

During re-operation after LTG, the cause of JJO after LTG was found to be adhesions between the jejunojejunostomy staple line and abdominal tissue. We consider that the cause of JJOs in our three cases were closure of the entry hole of the jejunojejunostomy with a knotless barbed suture and serosal to muscle layer suture reinforcement of the jejunojejunostomy. This type of closure probably resulted in excessive kinking of the jejunojejunostomy. We recently commenced using barbed sutures in order to reduce surgical duration. Barbed sutures offer a fast, secure, and effective alternative to conventional suture repair during laparoscopic surgery. However, cases of surgical complications, such as $\mathrm{SBO}$, associated with the use of barbed suture devices have been reported [11, 12]. Subsequently, since we encountered these three cases of JJOs over a short period of time, we reinstituted the traditional closure method of the entry hole using only continuous 3-0 Vicryl sutures. We have not encountered JJOs since then.

In a recent study of 1097 patients who underwent laparoscopic R-Y gastric bypass [3], early JJO occurred in 13 patients (1.2\%). In that report, the average time to presentation was 15 days (range 5-27). Patients presented with a combination of nausea, vomiting, and abdominal pain; all underwent $\mathrm{CT}$ to confirm the diagnosis. Ten patients $(77 \%)$ were treated conservatively and three $(23 \%)$ required operation with the creation of a new jejunojejunostomy between the distal Roux limb and proximal common channel to bypass the narrowed jejunojejunostomy.

In our three cases, all patients could be diagnosed by $\mathrm{CT}$ and upper gastrointestinal series. The average interval from gastrectomy to JJO was 5 days (range 2-7), initial clinical symptoms were vomiting in all three patients, and simultaneous upper abdominal pain in one patient. These results were similar to recent reports.

We conducted FBD in all three cases after unsuccessful conservative treatment using an ileus tube.

FBD resulted in rapid improvement in clinical symptoms, and all patients were able to avoid re-operation. The average period to FBD from JJO was 10 days (range 4-14). The average procedure time was $46 \mathrm{~min}$ (range 29-68), and average duration to oral intake was 4 days (range 2-5), The average hospital stay following FBD was 12 days (range 9-15). There were no complications. The longer procedure time $(68 \mathrm{~min})$ in case 1 was due to a longer time to guidewire passage.

Existing guidelines recommend that trials of nonoperative management should not exceed 3-5 days because the likelihood of spontaneous resolution decreases substantially after this period [13, 14]. However, in our experience, FBD was useful after conservative treatment for 14 days, suggesting that there might be no restriction on the timing for FBD for SBO in the absence of findings suggestive of complete obstruction, strangulation, or necrosis. 
The limitations of our research are that it included only few cases and only short-term follow-up was performed.

However, our experience suggests that FBD could be an alternative procedure to avoid surgery for ESBO after gastrectomy with R-Y reconstruction.

\section{Conclusion}

FBD could be a feasible procedure to avoid surgery for ESBO after gastrectomy with R-Y reconstruction.

\section{Abbreviations}

SBO: Small bowel obstruction; R-Y reconstruction: Roux-en-Y reconstruction: ESBO: Early small bowel obstruction; FBD: Fluoroscopic balloon dilation; JJO: Jejunojejunostomy obstruction; LDG: Laparoscopic distal gastrectomy; LTG: Laparoscopic total gastrectomy; RADG: Robot-assisted distal gastrectomy; RATG: Robot-assisted total gastrectomy

\section{Acknowledgements}

None.

\section{Authors' contributions}

TK: study design, data collection, data analysis, and writing of the manuscript. HO: critical revision of the manuscript. EY: critical revision of the manuscript. HT: Figure creation of the manuscript. YS: final approval of the article. All other authors: data collection. All authors read and approved the final manuscript.

\section{Funding}

The authors declare that they did not receive any funding for this report.

\section{Availability of data and materials}

Data sharing is not applicable to this article as no datasets were generated or analyzed during the current study.

\section{Ethics approval and consent to participate}

Not applicable.

\section{Consent for publication}

Written informed consent was obtained from the patients for publication of this case report and any accompanying images.

\section{Competing interests}

The authors declare that they have no competing interests.

\section{Author details}

'Department of Surgery, International University of Health and Welfare Hospital, 537-3, Iguchi, Nasushiobara, Tochigi 329-2763, Japan. ${ }^{2}$ Department of Radiology, International University of Health and Welfare Hospital, 537-3, Iguchi, Nasushiobara, Tochigi 329-2763, Japan.

Received: 27 March 2020 Accepted: 13 May 2020

Published online: 24 May 2020

\section{References}

1. Nelson L, Gonzales R, Haines K, Gallagher S, Murr M. Spectrum and treatment of small bowel obstruction after roux-en-Y gastric bypass. Surg Obes Relat Dis. 2006;2:337-83.

2. Husain S, Ahmed A, Johnson J, Boss T, O'Malley W. Small-bowel obstruction after laparoscopic roux-en-Y gastric bypass: etiology, diagnosis, and management. Arch Surg. 2007;142:988-93.

3. Lewis $C E$, Jensen $C$, Tejirian T, Dutson E, Mehran A. Early jejunojejunostomy obstruction after laparoscopic gastric bypass: case series and treatment algorithm. Surg Obes Relat Dis. 2009;5:203-7.

4. Cho M, Carrodeguas L, Pinto D, Lascano C, Soto F, Whipple O, et al. Diagnosis and management of partial small bowel obstruction after laparoscopic antecolic antegastric roux-en-Y gastric bypass for morbid obesity. J Am Coll Surg. 2006;202:262-8.
5. Gong JF, Zhu WM, Yu WK, Li N, Li JS. Conservative treatment of early postoperative small bowel obstruction with obliterative peritonitis. World J Gastroenterol. 2013;19:8722-30.

6. Sajja SB, Schein M. Early postoperative small bowel obstruction. Br J Surg. 2004;91:683-91.

7. Tsauo J, Kim KY, Song HY, Park JH, Jun EJ, Kim MT, et al. Fluoroscopic balloon dilation for treating postoperative nonanastomotic strictures. J Vasc Interv Radiol. 2017;28:1141-6.

8. Baars JE, Theyventhiran R, Aepli P, Saxena P, Kaffes AJ. Double-balloon enteroscopy-assisted dilation avoids surgery for small bowel strictures. World I Gastroenterol. 2017:23(45):8073-81.

9. Nakanishi K, Kanda M, Ito S, Mochizuki Y, Teramoto H, Ishigure K, et al. Propensity-score-matched analysis of a multi-institutional dataset to compare postoperative complications between Billroth I and roux-en-Y reconstructions after distal gastrectomy. Gastric Cancer. 2020. https://doi. org/10.1007/s10120-020-01048-6.

10. Inokuchi M, Kojima K, Yamada H, Kato K, Hayashi M, Motoyama K, et al. Long-term outcomes of roux-en-Y and Billroth-I reconstruction after laparoscopic distal gastrectomy. Gastric Cancer. 2013;16:67-73. https://doi. org/10.1007/s10120-012-0154-5.

11. Oor J, de Castro S, van Wagensveld B. V-loc ${ }^{T M}$ capable of grasping surrounding tissue causes obstruction at the jejunojejunostomy after rouxen-Y laparoscopic gastric bypass. Asian J Endosc Surg. 2015;8(2):209-11. https://doi.org/10.1111/ases.12169.

12. Jang SH, Jung YK, Choi SJ, Ha TK. Postoperative mechanical small bowel obstruction induced by V-Loc barbed absorbable suture after laparoscopic distal gastrectomy. Ann Surg Treat Res. 2017;92(5):380-2.

13. Maung AA, Johnson DC, Piper GL, Barbosa RR, Rowell SE, Bokhari F, et al. Evaluation and management of small-bowel obstruction: an eastern Association for the Surgery of trauma practice management guideline. J Trauma Acute Care Surg. 2012;73:S362-9.

14. Di Saverio S, Coccolini F, Galati M, Smerieri N, Biffi WL, Ansaloni L, et al. Bologna guidelines for diagnosis and management of adhesive small bowel obstruction (ASBO): 2013 update of the evidence-based guidelines from the world Society of Emergency Surgery ASBO working group. World I Emerg Surg. 2013;8:42.

\section{Publisher's Note}

Springer Nature remains neutral with regard to jurisdictional claims in published maps and institutional affiliations.

\section{Submit your manuscript to a SpringerOpen ${ }^{\circ}$ journal and benefit from:}

- Convenient online submission

- Rigorous peer review

- Open access: articles freely available online

High visibility within the field

- Retaining the copyright to your article

Submit your next manuscript at $\boldsymbol{\nabla}$ springeropen.com 